\title{
Editorial CEMRACS 2019
}

\author{
Geophysical Fluids \\ Gravity Flows
}

This special volume gathers research works that have been conducted during the 24th edition of the summer school CEMRACS (Centre d'Été Mathématique de Recherche Avancée en Calcul Scientifique), which took place at CIRM (Centre International de Rencontre Mathématique) in Luminy from July 15th to August 23rd, 2019. The CEMRACS, initiated in 1996 by Yvon Maday and Frédéric Coquel, is held every summer and consists in two parts : a summer school during the first week where several lectures, related to the topic of the CEMRACS, are given by leading scientists, and an intensive research session gathering young and senior researchers on projects proposed by academic scientists or industrials during the remaining five weeks.

The CEMRACS'19 was focused on the modelling, discretisation and simulation of fluid flows coming from problems in geophysics. This is a topic that becomes more and more crucial considering the increasing number of environmental issues like tropical cyclones, tsunamis, retreat of glaciers or even radioactive wastes management. Many works have already been dedicated to the study of geophysical fluid flows but efforts still need to be made in order to better understand and predict the evolution of these physical phenomena. Better models are derived by adding more physics in order to better approximate the complex dynamics of these flows. This added complexity makes the analysis of these models, the discretisation and the simulation more and more challenging. This volume of ESAIM:ProcS gathers some contributions on mathematical models, numerical schemes and their analysis in the geophysical fluids field. These contributions are the result of works conducted during the research session of CEMRACS'19. More details are available at http://smai.emath.fr/cemracs/cemracs19/ 
The organization of this edition of CEMRACS benefited from the support of AMIES, ANR, CEA, CentralSupélec, CNRS, EMS, ERC, GdR EGRiN, GdR MaNu, Institut de Mathématique de Bordeaux, Institut Camille Jordan, Institut D'Alembert, Institut Denis Poisson, Inria, INSA Toulouse, Labex Voltaire, LHSV / ENPC, Mésocentre Aix-Marseille, SMAI, Sorbonne Université, Université d'Orléans.

The organizers: Arnaud Duran, Benoit Fabrèges, Pauline Lafitte, Frédéric Lagoutière, Fabien Marche, Frédéric Rousset. 